Supplement of Hydrol. Earth Syst. Sci., 22, 5559-5578, 2018

https://doi.org/10.5194/hess-22-5559-2018-supplement

(C) Author(s) 2018. This work is distributed under

the Creative Commons Attribution 4.0 License.

(c) (1)

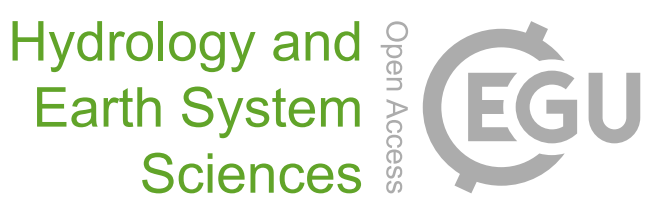

Supplement of

\title{
Evaluating and improving modeled turbulent heat fluxes across the North American Great Lakes
}

\section{Umarporn Charusombat et al.}

Correspondence to: Ayumi Fujisaki-Manome (ayumif@umich.edu)

The copyright of individual parts of the supplement might differ from the CC BY 4.0 License. 


\section{Supplemental materials}

Supplemental Table 1. The integrated forms of stability functions for momentum $\Psi_{M}$ in the five algorithms. $x$ and $x_{0}$ are defined as $x=$ $\left(1-\gamma_{m} \zeta\right)^{1 / 4}$ and $x_{0}=\left(1-\gamma_{m} \zeta_{0}\right)^{1 / 4}$, respectively.

\begin{tabular}{|c|c|c|c|}
\hline Algorithm name & \multicolumn{3}{|c|}{$\Psi_{M}$} \\
\hline & Unstable $(\zeta<0)$ & $\begin{array}{l}\text { Weakly stable }(0<\zeta \\
<1)\end{array}$ & Strongly stable $(\zeta>1)$ \\
\hline LS87 & $\begin{array}{c}2 \ln \left(\frac{1+x^{2}}{1+x_{0}^{2}}\right)-2\left(\operatorname{atan}\left(x^{2}\right)-\operatorname{atan}\left(x_{0}^{2}\right)\right), \\
\gamma_{m}=15 \\
\text { (Businger et al., 1971) }\end{array}$ & $\begin{array}{c}-4.7\left(\zeta-\zeta_{0}\right) \\
\text { (Holtslag et al., } 1990)\end{array}$ & $\begin{array}{l}-4.7\left(1-\zeta_{0}+\ln \zeta\right) \\
\text { (Holtslag et al., } 1990)\end{array}$ \\
\hline C89 & \multirow{3}{*}{$\begin{array}{c}2 \ln \left(\frac{1+x}{2}\right)+\ln \left(\frac{1+x^{2}}{2}\right)-2 \operatorname{atan}\left(x^{2}\right)+\frac{\pi}{2}, \\
\gamma_{m}=16 \\
\text { (Businger et al., 1971) }\end{array}$} & $\begin{array}{c}-5.2 \zeta \\
\text { (Holtslag et al., 1990) }\end{array}$ & $\begin{array}{c}-5.2(1+\ln \zeta) \\
(\text { Holtslag et al., } 1990)\end{array}$ \\
\hline Z98L & & $\begin{array}{c}-5 \zeta \\
\text { (Holtslag et al., 1990) }\end{array}$ & $\begin{array}{l}-4 \ln \zeta+5 \zeta_{0}-\zeta-4 \\
\text { (Holtslag et al., 1990) }\end{array}$ \\
\hline J99 & & \multicolumn{2}{|c|}{$\begin{array}{c}-[0.7 \zeta+0.75(\zeta-14.3) \exp (-0.35 \zeta)+10.7 \\
(\text { Beljaars and Holtslag, 1991) }\end{array}$} \\
\hline COARE & $\begin{array}{c}\Psi_{k}=\frac{\Psi_{k}+\zeta^{2} \Psi_{c}}{1+\zeta^{2}} \\
\Psi_{k}=2 \ln \left(\frac{1+x}{2}\right)+\ln \left(\frac{1+x^{2}}{2}\right)-2 \operatorname{atan}\left(x^{2}\right)+\frac{\pi}{2} \\
\text { (Businger et al., 1971), } \gamma_{m}=15 \\
\text { Convective behavior: } \\
\Psi_{c}=1.5 \ln \left(\frac{1+x_{c}+x_{c}^{2}}{2}\right)+\sqrt{3} \text { atan }\left(\frac{1+2 x_{c}}{\sqrt{3}}\right)+\frac{2 \pi}{\sqrt{3}}, \\
x_{0}=\left(1-\gamma_{c m} \zeta_{0}\right)^{1 / 3}, \gamma_{c m}=10.15 \\
(\text { Fairall et al., } 1996)\end{array}$ & \multicolumn{2}{|c|}{$\begin{array}{c}-\left[(1+\zeta)+\frac{2}{3}(\zeta-14.28) \exp (-0.35 \zeta)+8.525\right] \\
(\text { Beljaars and Holtslag, 1991) }\end{array}$} \\
\hline
\end{tabular}


Supplemental Table 2. The integrated forms of stability functions for temperature and humidity $\Psi_{\theta, q}$ in the five algorithms. $x$ and $x_{0}$ are defined as $x=\left(1-\gamma_{h} \zeta\right)^{1 / 4}$ and $x_{0}=\left(1-\gamma_{h} \zeta_{0}\right)^{1 / 4}$, respectively.

\begin{tabular}{|c|c|c|c|}
\hline Algorithm name & \multicolumn{3}{|c|}{$\Psi_{\theta, \mathrm{q}}$} \\
\hline & Unstable $(\zeta<0)$ & $\begin{array}{c}\text { Weakly stable }(0<\zeta \\
<1)\end{array}$ & Strongly stable $(\zeta>1)$ \\
\hline LS87 & $\begin{array}{c}2 \ln \left(\frac{1+x^{2}}{1+x_{0}^{2}}\right) \\
\gamma_{h}=9 \\
\text { (Businger et al., 1971) }\end{array}$ & $\begin{array}{l}-4.7 \operatorname{Pr}^{-1}\left(\zeta-\zeta_{0}\right) \\
\text { (Holtslag et al., } 1990)\end{array}$ & $\begin{array}{c}-4.7 \operatorname{Pr}^{-1}\left(1-\zeta_{0}+\ln \zeta\right) \\
\quad(\text { Holtslag et al., } 1990)\end{array}$ \\
\hline C89 & \multirow{3}{*}{$\begin{array}{c}2 \ln \left(\frac{1+x^{2}}{2}\right) \\
\gamma_{h}=16 \\
\text { (Businger et al., 1971) }\end{array}$} & $\begin{array}{c}-5.2 \zeta \\
\text { (Holtslag et al., 1990) }\end{array}$ & $\begin{array}{c}-5.2(1+\ln \zeta) \\
(\text { Holtslag et al., } 1990)\end{array}$ \\
\hline Z98L & & $\begin{array}{c}-5\left(\zeta-\zeta_{0}\right) \\
\text { (Holtslag et al., } 1990)\end{array}$ & $\begin{array}{l}-4 \ln \zeta+5 \zeta_{0}-\zeta-4 \\
\text { (Holtslag et al., 1990) }\end{array}$ \\
\hline J99 & & \multicolumn{2}{|c|}{$\begin{array}{c}-[0.7 \zeta+0.75(\zeta-14.3) \exp (-0.35 \zeta)+10.7] \\
(\text { Beljaars and Holtslag, 1991) }\end{array}$} \\
\hline COARE & $\begin{array}{c}\Psi_{k}=\frac{\Psi_{k}+\zeta^{2} \Psi_{c}}{1+\zeta^{2}} \\
\Psi_{k}=2 \ln \left(\frac{1+x^{2}}{2}\right) \text { (Businger et al., 1971), } \\
\text { Convective behavior: } \\
\Psi_{c}=1.5 \ln \left(\frac{1+x_{c}+x_{c}^{2}}{2}\right)+\sqrt{3} \text { atan }\left(\frac{1+2 x_{c}}{\sqrt{3}}\right)+\frac{2 \pi}{\sqrt{3}}, \\
x_{0}=\left(1-\gamma_{c h} \zeta_{0}\right)^{1 / 3}, \\
\gamma_{c h}=34.15 \\
\text { (Fairall et al., 1996) }\end{array}$ & \multicolumn{2}{|c|}{$\begin{array}{c}-\left[\left(1+\frac{2}{3} \zeta\right)^{1.5}+\frac{2}{3}(\zeta-14.28) \exp (-0.35 \zeta)+8.525\right] \\
(\text { Beljaars and Holtslag, 1991) }\end{array}$} \\
\hline
\end{tabular}




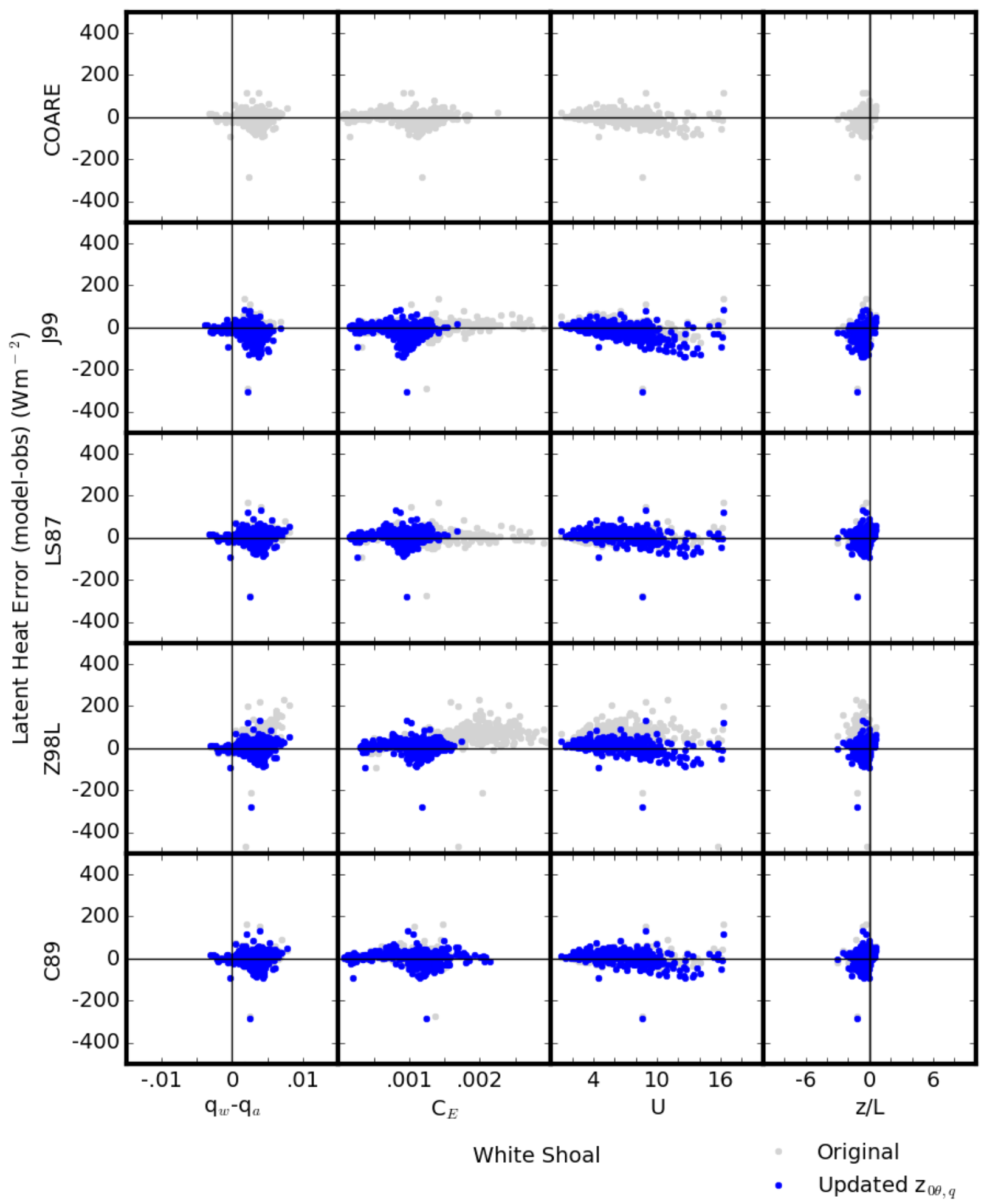

Figure S1. Errors of latent heat flux ( $y$-axis) versus specific humidity difference between water surface and air at the sensor height $q_{w}-q_{a}\left[\mathrm{~kg} \cdot \mathrm{kg}^{-1}\right]$, transfer coefficient $C_{E}[-]$, wind speed $U\left[\mathrm{~m} \cdot \mathrm{s}^{-1}\right]$, and stability factor $z / L(x$-axis) for the five algorithms at White Shoal. Each dot represents a daily mean value. Gray and blue dots indicate the results with the original and updated $z_{0 q}$ formulae, respectively. 


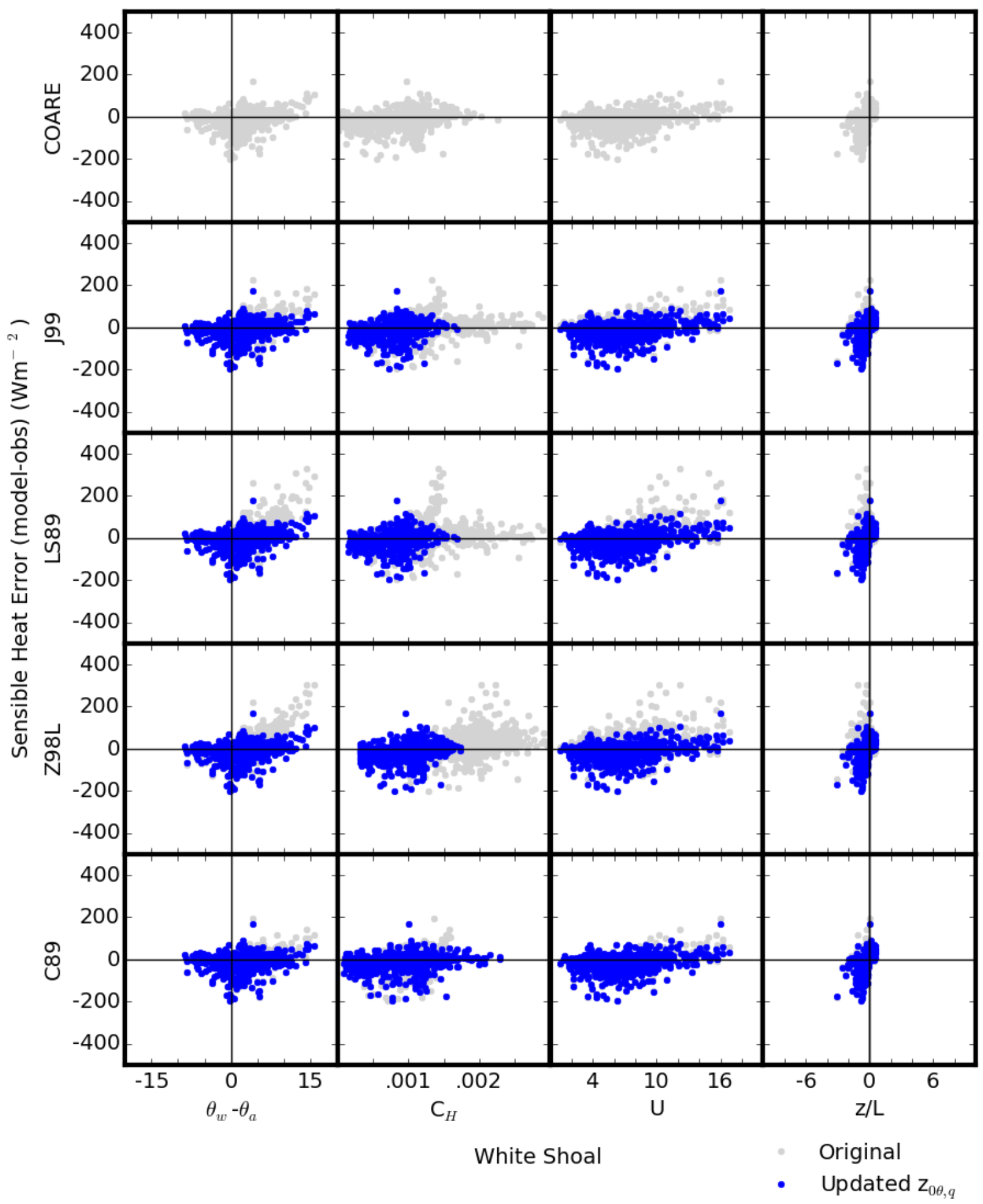

Figure S2. Errors of sensible heat flux (y-axis) versus temperature difference between water surface and air at the sensor height $\theta_{w}-\theta_{a}\left[{ }^{0} \mathrm{C}\right]$, transfer coefficient $C_{H}[-]$, wind speed $U\left(\mathrm{~m} \cdot \mathrm{s}^{-1}\right)$, and stability factor $z / L(x$-axis) for the five algorithms at White Shoal. Each dot represents a daily mean value. Gray and blue dots indicate the results with the original and updated $z_{0 \theta}$ formulae, respectively. 


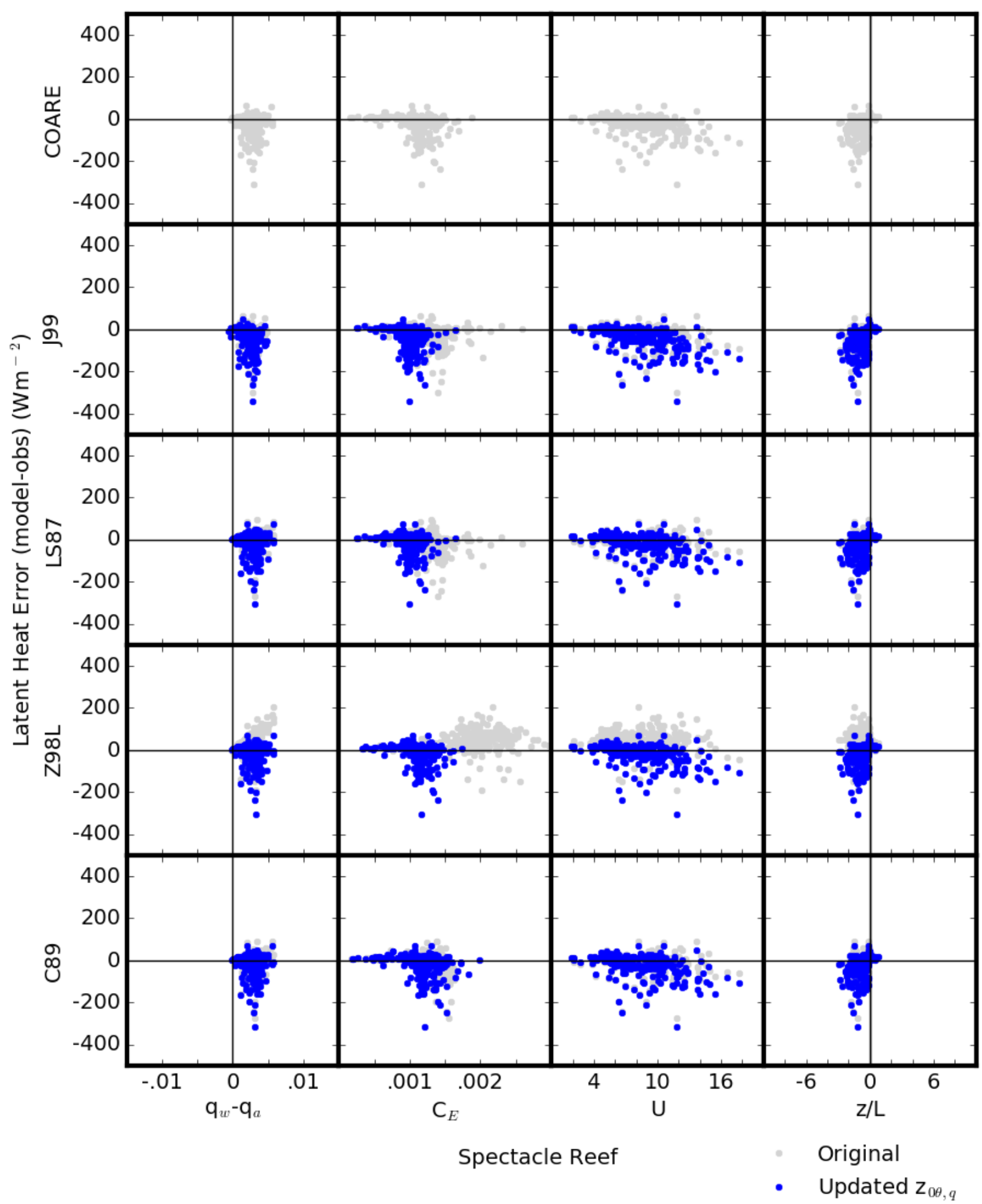

Figure S3. Similar to Fig. S1, but at Spectacle Reef. 


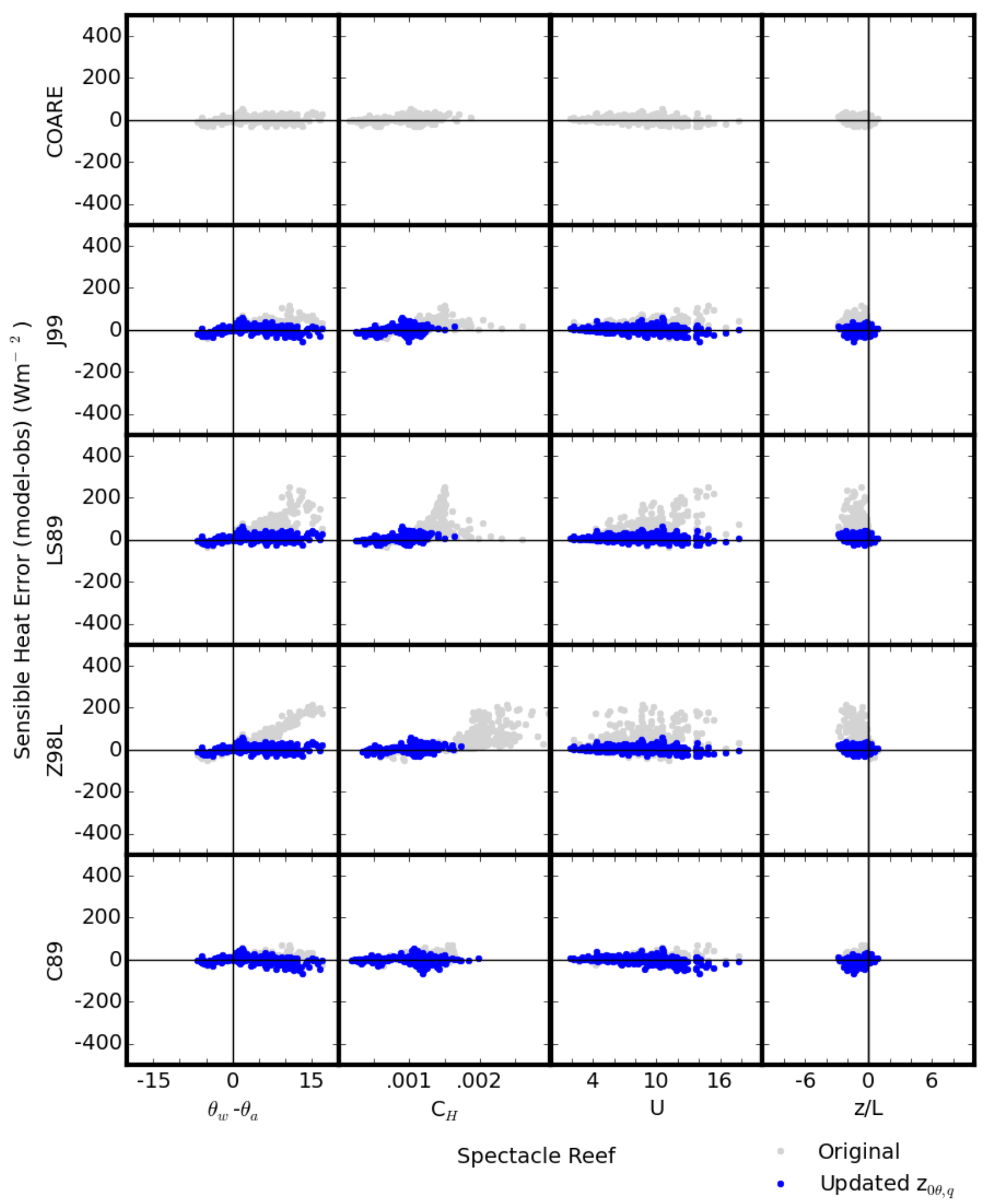

Figure S4. Similar to Fig. S2, but at Spectacle Reef. 


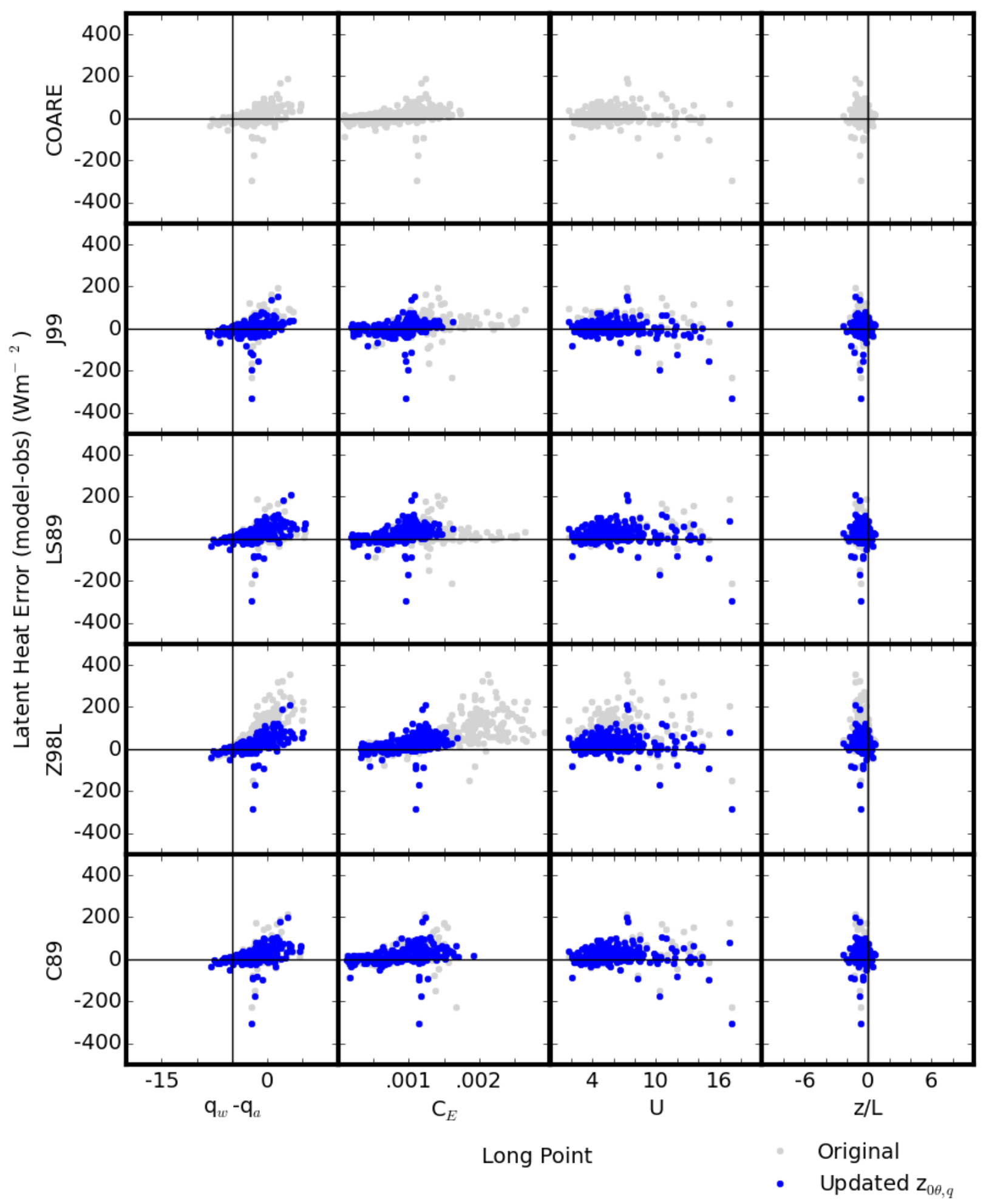

Figure S5. Similar to Fig. S1, but at Long Point. 


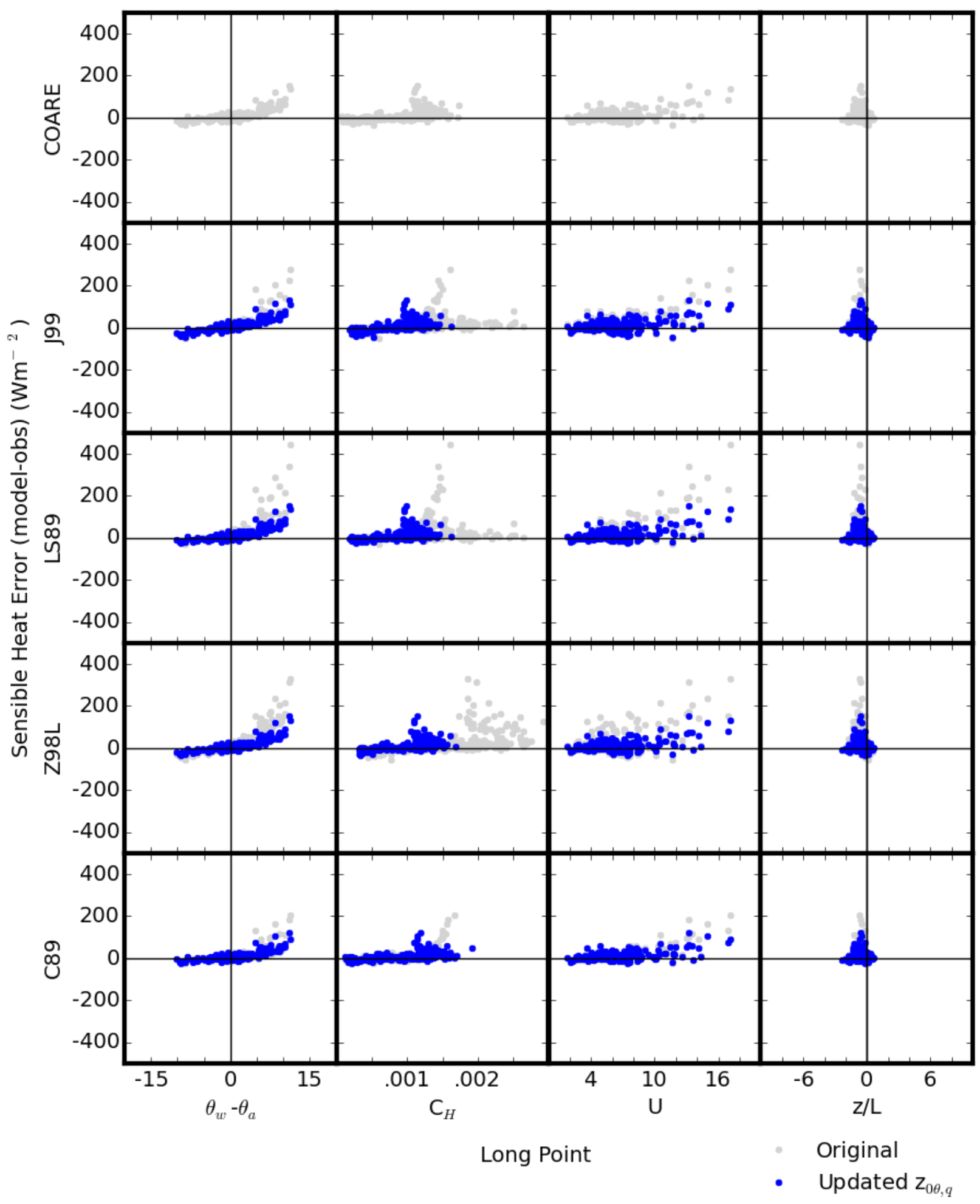

Figure S6. Similar to Fig. S2, but at Long Point. 


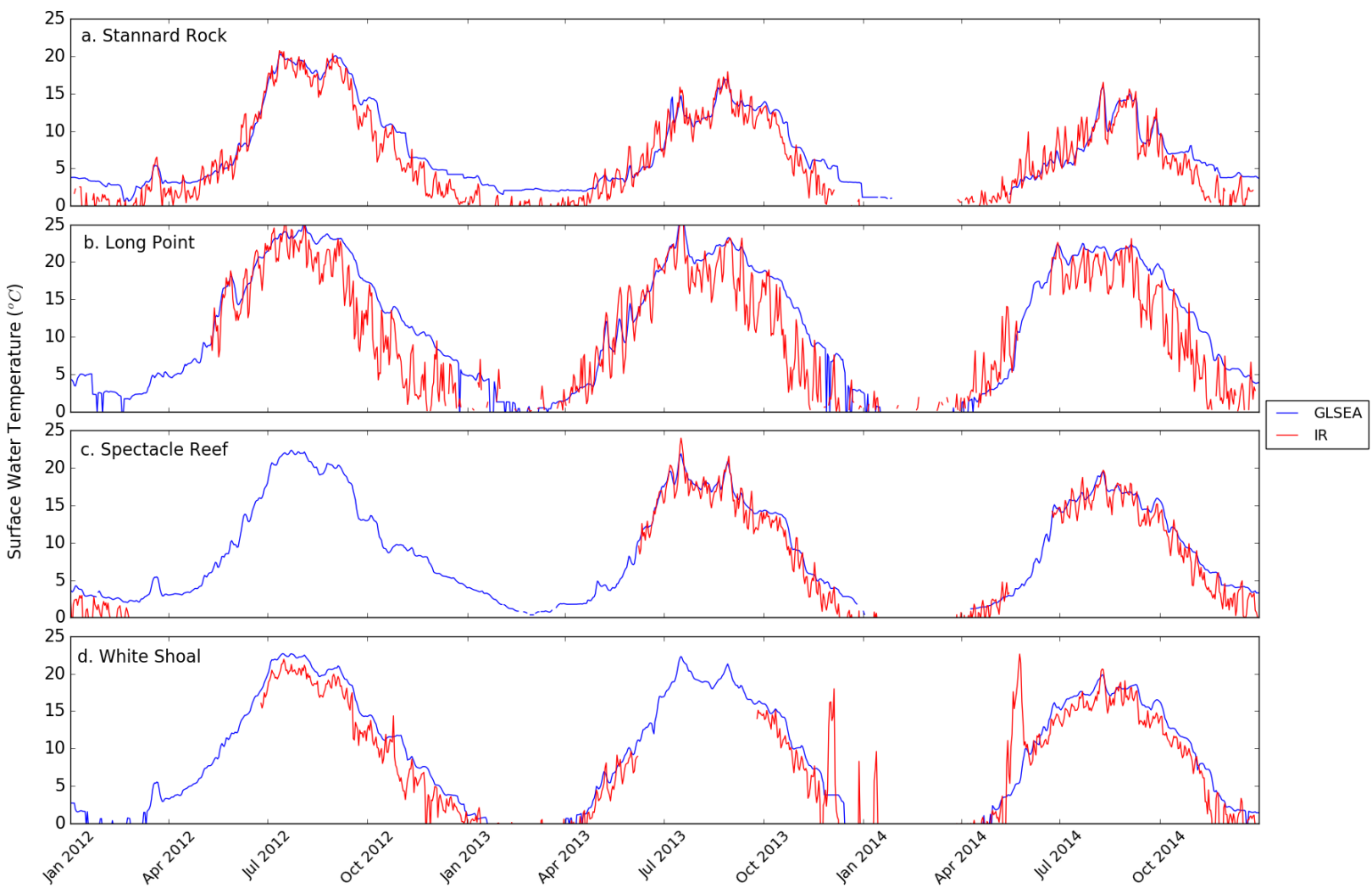

Figure S7. Daily water surface temperature at the four stations from GLSEA and Infrared thermometers (IRTs, Apogee IRR-T). For GLSEA, the closest pixel to each of the four stations was chosen. Values below zero are masked out. 